\title{
Acknowledgment of JAISE's referees
}

Over the past four years of its life, our Journal has been supported by a large number of colleagues who contributed with their time and expertise to assess the quality of the submissions to JAISE and helped decide which papers qualify to be published. These reviewers and referees are a very important part of the JAISE community and we would like to explicitly thank all of them for their valuable contributions.

The effort of reviewers often remains unnoticed in the community served by a journal, especially in a blind review system. From this year, we start the practice of acknowledging the participation of our reviewers in the making of JAISE.

As a second step towards making our gratitude explicit and highlighting the importance of the contributions made by our reviewers, we will also implement the practice of selecting two reviewers who have consistently provided detailed and quality reviews and inviting them to serve as part of the Editorial Board of JAISE.

The list of reviewers in the period 2009-2012 is ${ }^{1}$ : Agnar Aamodt, Andrea Abate, Giovanni Acampora, Dr. Toufik Ahmed, Marco Aiello, Abdullah Al Mahmud, Dzmitry Aliakseyeu, Colin Allison, Yiannis Aloimonos, Ricardo Alonso, Chen An, Elisabeth André, Cecilio Angulo, Ville Antila, Grigoris Antoniou, Masahiro Araki, Oya Aran, Bert Arnrich, Eleana Asimakopoulou, Vassilis Athitsos, Daniel Avrahami, Asier Aztiria, Sebastian Bader, Javier Bajo, Erwin Bakker, Matthias Baldauf, Matt Ball, Ana Alice Baptista, Jakob Bardram, Payam Barnaghi, Joao Barreto, João Barroso, Nikos Batalas, Julia Beamish, Michael Beigl, Yacine Bellik, Jérôme Berclaz, Marc Berenguer, Keni Bernardin, Ana Bernardos, Tim Bickmore, Antonis Bikakis, Jit Biswas, Alan Blackwell, Juan Blaya, Marco Blumendorf, Olivier Boissier, Dario Bonino, Tibor Bosse, Juan Botia, Sabri Boughorbel, Abdenour Bouzouane, Patrick Brezillon, Will Bridewell, Gregor Broll, Murtaza
Bulut, David Burden, Alberto Caballero, Giacomo Cabri, Victor Callaghan, Charles Callaway, Jiannong Cao, Javier Carbó, Jörg Cassens, Federico Castanedo, Luis Castro, Dan Chalmers, Yiqiang Chen, Louis Chen, Kefei Chen, Lorenzo Chiari, Minsu Cho, Dongkyu Choi, Hao Chu, Maurice Chu, Sook-Ling Chua, Laurence Claeys, Adrian Clear, Martin Colley, Juan Corchado, Antonio Coronato, Stefania Costantini, Lynne Coventry, Flavio da Silva, Dragos Datcu, Scott Davidoff, Marc Davies, Debraj De, Francisco De Paz, Boris De Ruyter, Liyanage De Silva, Ramon Delgado, Flávia Delicato, Anind Dey, Cem Direkoglu, Monica Divitini, Patrícia Dockhorn Costa, Jeyoun Dong, James Dooley, Olivier Dousse, Erik Dovgan, Thomas Drugman, Hakan Duman, Schahram Dustdar, Bruce Edmonds, Henk Eertink, Simon Egerton, Engin Erzin, Mbou Eyole-Monono, Babak Farshchian, Alejandro Fdez, Florentino FdezRiverola, Rune Fensli, Antonio Fernández Caballero, Lino Figueiredo, Simone Fischer-Huebner, Mary Ellen Foster, Chris Fowler, Lutz Frommberger, Kary Främling, Li-Chen Fu, Yongji Fu, Rubén Fuentes, Kaori Fujinami, Francesco Furfari, Óscar García, Manuel Garcia-Herranz, Michael Gardner, Chris Geib, Gary Geumbae Lee, Theo Gevers, Tomas Gillo, Owen Gilson, Andreas Girgensohn, Sylvain Giroux, Hristijan Gjoreski, Karl Goeschka, Carles Gomez, Bjoern Gottfried, Christos Goumopoulos, Dimitris Grammenos, Ovidiu Grigore, Zahia Guessoum, Christian Guetl, Ning Gui, Bin Guo, Micha Haas, Hani Hagras, Karen Zita Haigh, Klaus Hansen, Vuokko Harma, Béatrice Hasler, Mark Hawley, Pablo Haya, Tamara Hayes, Tobias Heinroth, Rim Helaoui, Robin Hess, Dirk Heylen, Cristian Hofmann, HansJuergen Holland, Paul Holleis, Anne Holohan, Seongsoo Hong, Mark Hoogendoorn, Bernard Horan, Jane Hunter, Tim Hussein, Dr. Lazaros Iliadis, Kentaro Ishii, Alex Jaimes-Larrarte, Vikramaditya Jakkula, Say Jang, Juan JimenezGarcia, Michael John, Brian Johnson, Kristiina Jokinen, P. Jonker, Hussein

\footnotetext{
${ }^{1}$ Names appear as they have been entered in the online peer review system.
} 
Joumaa, Vicente Julián, Eija Kaasinen, Bostjan Kaluza, Achilles Kameas, Felix Kamieth, Charalampos Karagiannidis, Georgios Karagiannis, Henry Kautz, Fahim Kawsar, Shimura Kenichiro, Peter Kiefer, Inchul Kim, Thomas Kirste, Michel Klein, Thomas Kleinberger, Kamil Kloch, Hoon Ko, Bastian Koenings, Mario Kolberg, Andreas Komninos, Savas Konur, Joke Kort, simon Kozina, jana Krivec, Ben Krose, Kai Kunze, Tei Laine, Josef Langer, Freddy Lecue, Seok-Won Lee, Minsoo Lee, Youngho Lee, Ronald Leenes, Enrique Leon, James Lester, Qun Li, Mei Yii Lim, Luís Lima, Kay Lindecrantz, Chengzhi Liu, Jun Liu, Daniel Livingstone, Guillaume Lopez, Ahmad Lotfi, Manolis Lourakis, Artur Lugmayr, Paul Lukowicz, Mitja Lustrek, Damian Lyons, José Machado, Nadia Mana, Francisco Maranhão, Domen Marincic, Goreti Marreiros, Natividad Martinez Madrid, Fulvio Mastrogiovanni, Paul McCullagh, Michael McTear, Vassilis Megalooikonomou, Alexander Meschtscherjakov, Florian Michahelles, Wolfgang Minker, Dorothy Monekosso, Simone Mora, Ramon Morros, Claude Moulin, Andrés Muñoz, Jani Mäntyjärvi, Tommi Määttä, Tatsuo Nakajima, Jin Nakazawa, Ali Nazari, Anton Nijholt, Paulo Novais, Petteri Nurmi, John O'Donoghue, Michael O'Grady, Ard Oerlemans, J. Ortega, Katsunori Oyama, José Palma, Giorgio Panin, Koosha Paridel, David Pautler, Dana Pavel, Wen-Chih Peng, Elizabeth Perkins, Camille Persson, Rok Piltaver, Michael Pirker, Steve Pitchers, Thomas Ploetz, Johan Plomp, Matthijs Pontier, Emanuel Popovici, Ronald Poppe, Eric Postma, Andrea Prati, Davy Preuveneers, Gilles Privat, Hervé Provost, Juhua Pu, Boon Kiat Quek, Wasim Raad, Nitendra Rajput, Carlos Ramos, Fano Ramparany, Anand Ranganathan, Parisa Rashidi, Cyril Ray, Madhu Reddy, Paolo Remagnino, Gunther Retscher, Daniele Riboni, Till Riedel, Jukka Riekki, Jarno Riistama, Fernando Rivera-Illingworth, Valen- tin Robu, Fátima Rodrigues, Marcela Rodriguez, Marc Roelands, Teresa Romão, Natalia Romero, Thomas Roth-Berghofer, George Roussos, Enrico Rukzio, Mohd Mohamad, Albert Salah, Jorge Santos, Ichiro Satoh, Lauri Savioja, Gregor Schiele, Bernt Schiele, Maximilian Schirmer, Andreas Schmidt, Hedda Schmidtke, Andreas Schrader, Nicu Sebe, Emilio Serrano, Caifeng Shan, Alexei Sharpanskykh, Feng Shu, Stephan Sigg, Nuno Silva, Bengt Arne Sjoqvist, Lauro Snidaro, John Soldatos, Ioannis Stamatiou, Kostas Stathis, Eleni Stroulia, Vitomir Struc, Peter Sun, Tomas Svoboda, Michal Szczerbak, Jürgen Tacken, Chantal Taconet, Gabriel Takacs, Kazunori Takashio, Dante Tapia, Teck-Hou Teng, Moritz Tenorth, Sotirios Terzis, Yoshito Tobe, Behcet Toreyin, An Tran, Jan Treur, Tuomo Tuikka, Aitor Urbieta, Laura Vadillo, Miika Valtonen, Jakob van de Laar, Betsy Van Dijk, Joy Van Helvert, Kristof Van Laerhoven, Jukka Vanhala, Yves Vanrompay, Bart Vanrumste, Eloisa Vargiu, Martijn Vastenburg, Jo Vermeulen, Alessandro Vinciarelli, A. Visser, Eric Wade, Daniel Wagner, Wolfgang Wahlster, Chenliang Wang, Lisanne Warmerdam, Michael Weber, Zhigang Wen, Janet Wesson, Geoff West, Reiner Wichert, Alexander Wiethoff, Michael Wittke, David Wortley, Hejun Wu, Chen Wu, Jianxin Wu, Yanwei Wu, Nigel Wynne, Qingjun Xiao, Poonam Yadav, Tatsuya Yamazaki, Jungjin Yang, Xiaogang Yang, Allen Yang, Jaeyoung Yang, Ansar Ul Haque Yasar, Neil Yorke-Smith, Michael Youngblood, Marielba Zacarias, Ioannis Zaharakis, Carolina Zato, Huiru Zheng, Vincent Wenchen Zheng, Yin Zhu, Brian Ziebart, Vale Zita, and Elly Zwartkruis.

\footnotetext{
JAISE Editors-in-Chief Juan Carlos Augusto Hamid Aghajan
} 